\title{
Expression in transgenic mice of two genes of different tissue specificity integrated into a single chromosomal site
}

\author{
Paz Einat, Yehudit Bergman, ${ }^{1}$ David Yaffe, and Moshe Shani \\ Department of Cell Biology, The Weizmann Institute of Science, Rehovot, Israel ${ }^{1}$ The Hubert H. Humphrey Center for \\ Experimental Medicine and Cancer Research, Hadassah Medical School, Jerusalem, Israel
}

\begin{abstract}
Transgenic mice were used to study the expression of pairs of genes with distinctly different tissue specificities, covalently linked and integrated into the same chromosomal site. A transgenic strain carrying, in close proximity and in the same orientation, the rat fast skeletal muscle myosin light-chain 2 (MLC2) gene and the mouse rearranged immunoglobulin $\kappa$ light-chain gene expressed the immunoglobulin gene specifically in the lymphoid tissues, whereas rat MLC2 transcripts were found in skeletal muscle but not in the spleen or the other tissues that were tested. In another transgenic strain, carrying the rat MLC2 gene and a modified rat skeletal muscle actin gene (actin-globin chimeric gene), transcripts of the rat MLC2 gene were detected in skeletal muscle only, whereas the actin-globin transcripts were detected in skeletal muscle as well as in the heart. Moreover, the expression of the chimeric gene was also developmentally regulated. Expression was higher in cardiac muscle than in the skeletal muscle of neonatal mice, whereas expression was higher in skeletal muscle in adult mice. This pattern is consistent with the regulation of the expression of the endogenous skeletal muscle actin gene. Thus, in those transgenic strains that expressed both genes, each gene retained its tissue specificity, in spite of their close proximity. These results indicate a high degree of autonomy of the control elements included in the cloned genomic DNA fragment and demonstrate that a single chromosomal site can be permissive for the proper expression of two genes with different tissue specificities.
\end{abstract}

[Key Words: Gene expression; gene transfer; transgenic mice; single chromosomal site; myosin light-chain 2 gene; actin-globin chimeric gene; immunoglobulin $\kappa$ gene]

Received August 10, 1987; revised version accepted October 12, 1987.

DNA-mediated gene transfer experiments reveal a wide variation in the expression of exogenous genes among various clones of transfected cells (Renkawitz et al. 1982; Chao et al. 1983; Kondoh et al. 1983; Charnay et al. 1984; Melloul et al. 1984). Similar quantitative variations in the levels of gene expression are commonly observed in transgenic mice. More interestingly, qualitative stably inherited differences in gene expression have been observed among transgenic mouse strains produced by microinjection of identical gene constructs (Babinet et al. 1985; Chada et al. 1985; Shani 1985, 1986; Townes et al. 1985a; Hammer et al. 1986; Shani and Yaffe 1986). These variations were attributed to the effects of the host DNA sequences at the site of integration on the expression of the exogenous genes. This notion is further supported by a number of cytogenetic and genetic studies showing chromosomal position effects on gene expression. A classic example of a position effect occurs in Drosophila, where the white mutant is produced by a chromosome rearrangement in which a gene for eye color is translocated from its normal euchromatic envi-

'Present address: Institute of Animal Sciences, ARO, The Volcani Center Bet-Dagan 50-250, Israel. ronment to the vicinity of a heterochromatic region (Spofford 1976). A similar phenomenon is also observed in mice when autosomal loci are inactivated due to translocation into the heterochromomatic $X$ chromosome (Cattanach 1974). Another example of a chromosomal position effect is the formation of tissue-specific tumors associated with translocations of proto-oncogenes (reviewed in Klein and Klein 1985).

Gene transfer into the mouse germ line provides a useful tool for analyzing the interrelation between a gene and its immediate DNA environment on its expression in various tissues. As an approach to studying the possible cis-acting effects of the DNA environment on the expression of the inserted gene, we have microinjected pairs of well-characterized cloned genes of differing tissue specificities, which were previously covalently linked, thus bringing them into close proximity. Introduction of such constructs into the germ line enables the comparison of their expression in many tissues following a single integration event. In the present communication, we describe experiments in which the rat fast skeletal muscle myosin light-chain 2 (MLC2) gene was introduced into the mouse germ line on a plasmid DNA also containing either the rearranged mouse im- 
munoglobulin $\kappa$ light-chain gene or a chimeric actinglobin gene. We have shown previously that the exogenous rat MLC2 gene is specifically expressed in skeletal muscle, whereas the chimeric actin-globin gene is expressed in skeletal, as well as in cardiac, muscles of transgenic mice (Shani 1985, 1986; Shani and Yaffe 1986). Brinster et al. (1983) have shown that the $\kappa$ immunoglobulin gene is specifically expressed in the spleen. Our results with the two pairs of genes showed that in those transgenic strains in which both genes were expressed, each gene retained its tissue specificity, indicating a high degree of autonomous behavior of the control sequences included in the cloned gene DNA fragment.

\section{Results}

Production of transgenic mice carrying the rat MLC2 gene linked to the mouse rearranged immunoglobulin $\kappa$ light-chain gene

The structure of plasmid pMLC-k, carrying the two genes, is shown in Figure 1. A 7.0-kb EcoRI-BamHI DNA fragment containing the rearranged mouse $\kappa$ lightchain gene from the myeloma cell line MOPC41 (Bergman et al. 1984) was inserted into the SalI site of plasmid $\mathrm{pMLCH}$, containing the entire rat MLC2 gene in a 4.8-kb DNA fragment (Nudel et al. 1984), so that both genes were present in the same transcriptional orientation. The resultant $16-\mathrm{kb}$ plasmid was linearized by a single NdeI cut within the pBR322 vector before injection. Approximately 100 molecules of DNA were microinjected into the pronucleus of fertilized eggs, as described (Shani 1985). A total of 80 microinjected eggs were transferred into the oviducts of four pseudopreg-

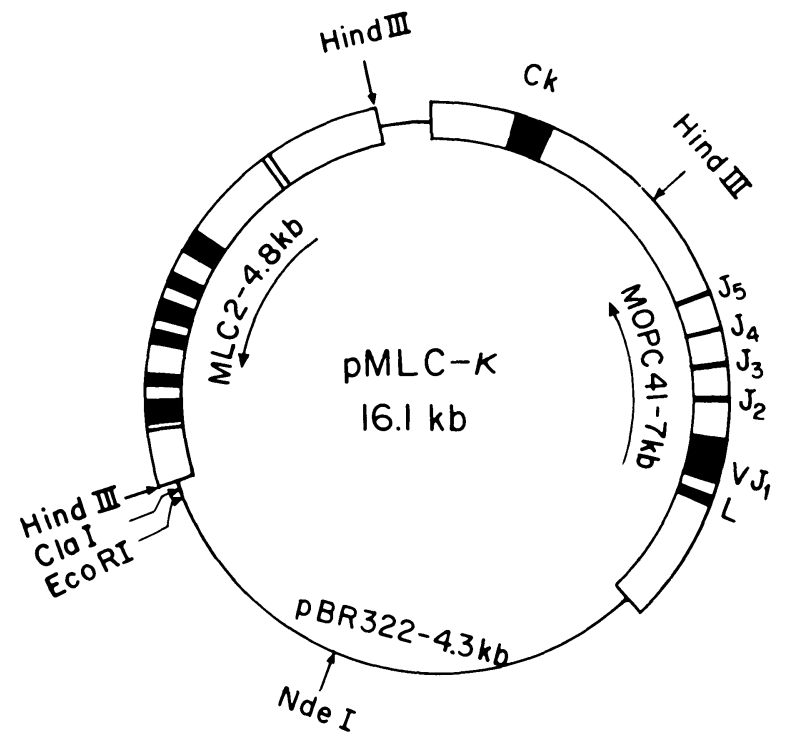

Figure 1. Map of plasmid pMLC-k. The 7.0-kb EcoRI-BamHI fragment containing the rearranged mouse immunoglobulin $\kappa$ light-chain gene was inserted into the SalI site of plasmid $\mathrm{pMLCH}$, containing the rat MLC2 gene, as described in Materials and methods. The solid boxes represent exons, and the open boxes represent introns and flanking DNA sequences. Direction of transcription is indicated by arrows. nant CD-1 females. Of the 15 mice born, three contained the injected DNA.

\section{Expression of the rat MLC2 and the immunoglobulin genes}

RNA was prepared from skeletal muscle, spleen, and other organs of the progeny of the three transgenic mice and assayed for the amount of $\kappa$ or MLC2 mRNA. The presence of immunoglobulin gene transcripts in these RNA preparations was determined by the S1 endonuclease assay using the 180-bp PvuI-BstNI DNA fragment labeled in the leader exon as a probe (Fig. 2). Spleen RNA from the three transgenic mice and thymus RNA from mice LCk3 and LCk4 protected the diagnostic 80nucleotide DNA fragment. The level of expression of the microinjected $\kappa$ gene in the spleen was about fourfold higher than that in the thymus. No $\kappa$ transcripts could be found in thymus RNA of transgenic strain LCk5. Because thymus preparations are known to be easily contaminated with parathymic lymph nodes (Storb et al. 1984), it is possible that the low level of transcripts found in the thymus of two of these mice reflects a contamination with these lymph node cells. No $\kappa$ transcripts were detected in skeletal muscle RNA from any of the mice. $\kappa$ mRNA was not detected in spleens of normal mice and transgenic mice containing other exogenous genes, except for a scarcely detectable level of hybridization with RNA from the spleen of mouse $\mathrm{CV}_{2}$ (a transgenic strain carrying the chimeric actin-globin gene). A similar finding was reported by Storb et al. (1984), using another $\kappa$ gene probe and normal mouse spleen RNA.

The presence of rat MLC2 transcripts in the RNA preparations was determined by the RNase protection assay, using a uniformly ${ }^{32}$ P-labeled complementary SP6 RNA probe derived from the $5^{\prime}$ region of the rat MLC2 gene (see Fig. 3). Hybridization of the probe with RNA prepared from rat skeletal muscle protected a 50-nucleotide fragment (Fig. 3). No rat transcripts could be detected in skeletal muscle or any of the other tissues of transgenic mice LCk3 and LCk5. In mouse LCk4, however, transcripts were detected in skeletal muscle but not in spleen or the other tissues tested (Fig. 3). Thus, in this transgenic mouse strain, the rat MLC2 gene was specifically expressed in skeletal muscle, despite the close physical association with the strong control elements of the immunoglobulin gene.

\section{Linkage between the exogenous rat MLC2 and the immunoglobulin genes in the genome of the transgenic strain LCk4}

As both the immunoglobulin and the MLC2 genes were expressed and retained their tissue specificity in the transgenic mouse strain LCk4, it was important to determine whether the exogenous genes were still linked and integrated in a single chromosomal site in this strain. Figure 4 shows a Southern blot analysis of the linkage between the two genes in genomic DNA of the three transgenic strains. High-molecular-weight tail DNA samples were digested with the restriction en- 


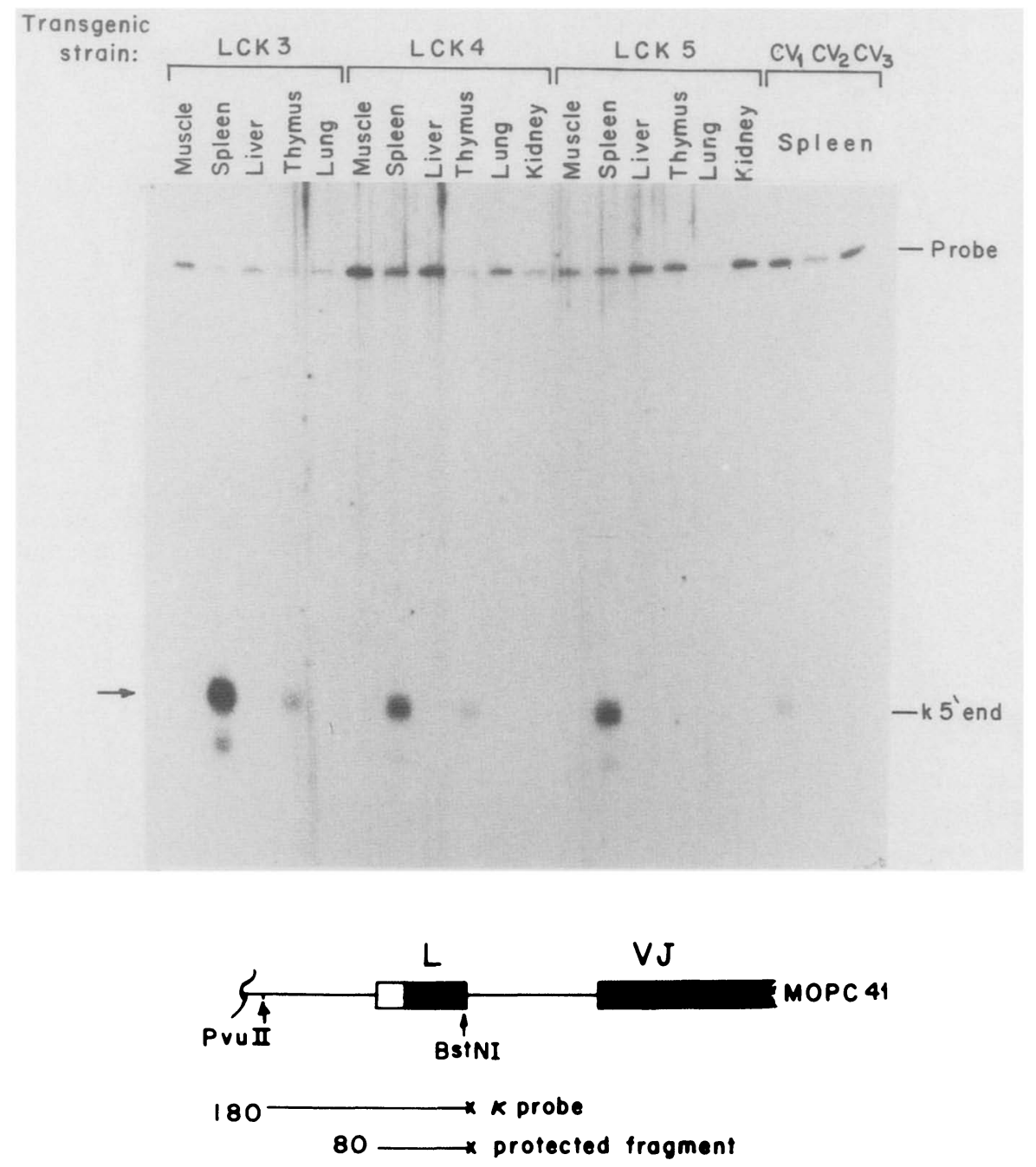

Figure 2. S1 analysis of $\kappa$ gene transcripts in various tissues of transgenic mice carrying plasmid, pMLC-k. Total RNAs (10 $\mu \mathrm{g}$ ) from the indicated tissues of transgenic mice were hybridized with a $5^{\prime}$-end-labeled 180-bp PvuII-BstNI fragment derived from the $5^{\prime}$ region of the $\kappa$ gene. Spleen RNAs from transgenic mice carrying the chimeric actin-globin gene (CV1, CV2, CV3) were used as controls. The labeled fragment protected by the exogenous gene transcripts is indicated by an arrow. The minor band found under the 80-nucleotide protected fragment probably resulted from overdigestion by the S1 nuclease. (Bottom) (Solid boxes) Immunoglobulin coding sequences; (open box) the 5'-untranslated region. The $5^{\prime}$ label is indicated by an asterisk. The numbers indicate the lengths of the probe and the protected fragments (nucleotides).

zymes BamHI, BglII, or HindIII, fractionated by gel electrophoresis, and blot-hybridized with either the $1-\mathrm{kb}$ DNA probe derived from the $5^{\prime}$ end of the rat MLC2 gene (probe I) or the $7.0-\mathrm{kb}$ DNA fragment carrying the rearranged immunoglobulin gene (probe II).

HindIII cuts twice at the ends of the MLC2 gene and once within the $\kappa$ gene. Hybridization with the MLC2 probe reveals the expected 4.8 - and $8.0-\mathrm{kb}$ bands of the exogenous and endogenous MLC2 genes, respectively, in the genome of strain LCk4. The faint band of $7.9 \mathrm{~kb}$ is a result of contamination of the probe with $\mathrm{pBR}$ sequences. The $\kappa$ probe reveals the expected 7.9- and 3.5$\mathrm{kb}$ bands of the exogenous genes and the $2.7-$ and $4.5-\mathrm{kb}$ bands of the endogenous genes. The number of copies of the integrated DNA was determined by comparing the intensities of the endogenous and exogenous $\kappa$-gene bands. Thus, strain LCk4 carries two to three copies per diploid genome.
BglII cuts at two adjacent sites in the MLC2 gene and at two sites, $2.8 \mathrm{~kb}$ apart, in the $\kappa$ gene. A $2.6-\mathrm{kb}$ fragment, containing $5^{\prime}$ sequences of the MLC2 gene and 3' sequences of the $\kappa$ gene and $\mathrm{pBR}$ sequences, is expected and is indicative of the integrity of linkage between the two genes. The MLC2 probe reveals only this band in the genome of strain LCk4. The $\kappa$ probe also reveals this band, just under the intense $2.8-\mathrm{kb}$ band of the exogenous and the endogenous genes. In addition, it hybridizes with the $4.1-$ and the $7.2-\mathrm{kb}$ bands of the endogenous $\kappa$ genes and the $10.7-\mathrm{kb}$ fragment of the exogenous gene, which is expected from a head-to-tail orientation of the integrated copies. A $9.2-\mathrm{kb}$ band, indicative of head-to-head integration, is found in strains LCk3 and LCk5, and a band of $11.8 \mathrm{~kb}$, indicative of tail-to-tail integration, is found in strain LCk3.

BamHI cuts once within the vector DNA. A fragment of $16 \mathrm{~kb}$ (the original plasmid size) is expected if the mi- 


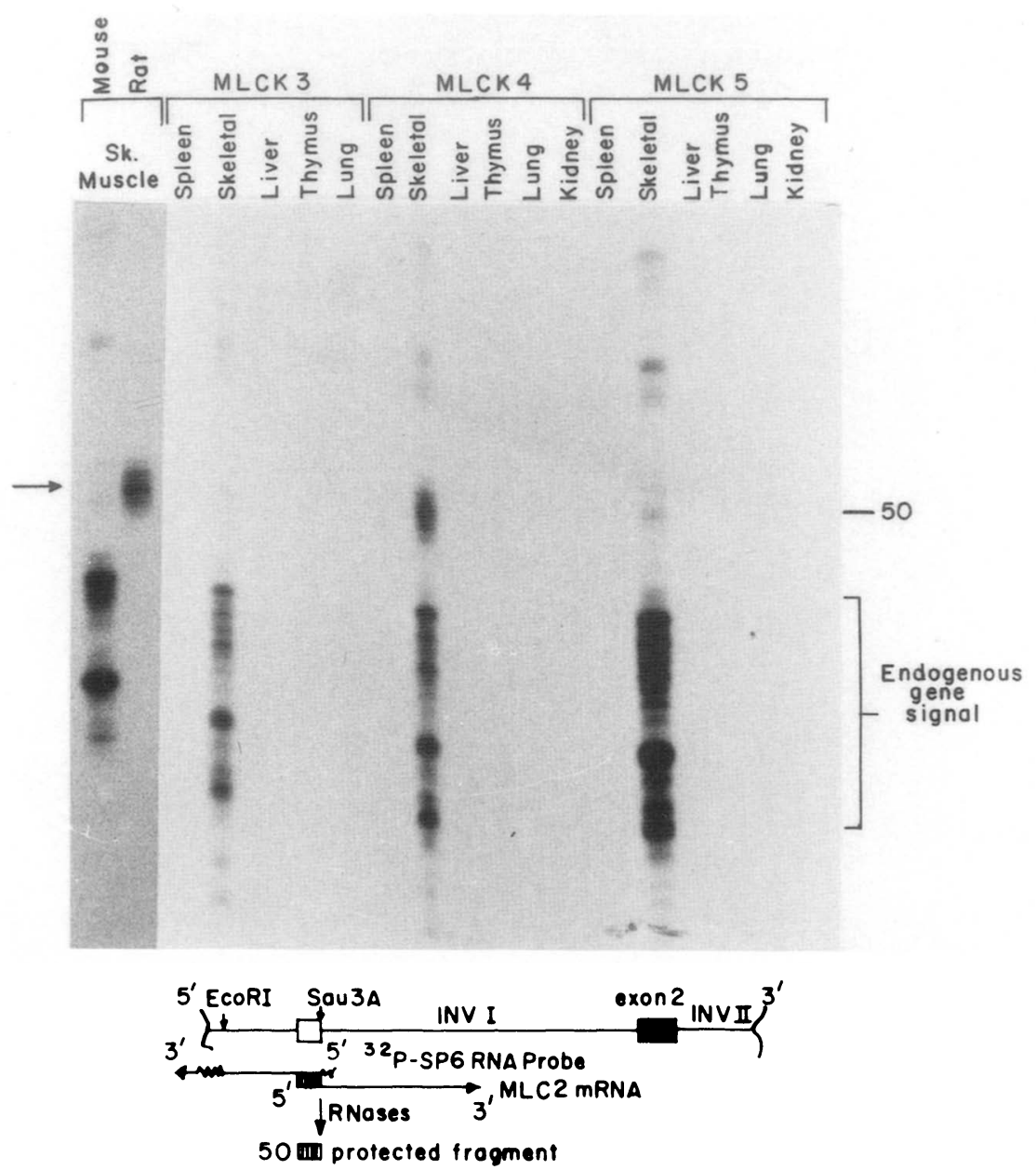

Figure 3. RNase mapping of the rat MLC2 transcripts in various tissues of transgenic mice carrying pMLC-k. Total RNAs from the indicated tissues of transgenic mice and from skeletal muscle of normal rat and mouse were hybridized with uniformly labeled complementary SP6 RNA probe derived from the $5^{\prime}$ region of the rat MLC2 gene. The protected fragment of 50 nucleotides results from specific hybridization to the rat MLC2 mRNA, whereas the products of cross-hybridization with the endogenous gene yield discrete bands of smaller sizes (Shani 1985; Shani and Yaffe 1986). The apparent signal in transgenic strain LCk5 in the 50 bp is due to cross-hybridization with the endogenous gene transcripts and is also detectable in an appropriate exposure of the control. The labeled fragment protected by the exogenous gene transcripts is indicated by an arrow. (Bottom) The probe was synthesized from an EcoRISau3A DNA fragment of the rat MLC2 gene cloned in the vector SP6. (Solid box) First coding exon of the rat MLC2 gene; (open box) 5' untranslated exon.

croinjected DNA was integrated in head-to-tail orientation, whereas integration in head-to-head or head-to-tail orientations should produce fragments of $18 \mathrm{~kb}$ or $14 \mathrm{~kb}$, respectively. In agreement with the results of the BglII digest, strain LCk4 reveals only the 16-kb band of the head-to-tail orientation, strain LCk5 reveals also the head-to-head orientation, whereas in strain LCk3 all three orientations exist. The BamHI digest also reveals the junction fragments with the host DNA. One minor band (marked by arrow) appears in each strain after hybridization with the MLC probe, and a different minor band appears in strains LCk3 and LCk4 after hybridization with the $\kappa$ probe. These results are consistent with a single site of integration of the microinjected DNA in all three strains. For strain LCk4 this was supported by pedigree analysis, showing that all the integrated DNA is found on one chromosome (data not shown).

The results using three different restriction enzymes show that in strain LCk4, two to three copies of the plasmid had integrated in one chromosomal site, all of them in the same orientation. No visible rearrangements could be detected.

\section{Production of mice carrying the rat MLC2 and the chimeric actin-globin genes}

The structure of plasmid CV-LC, containing the rat MLC2 gene juxtaposed to a chimeric actin-globin gene (pCV-LC), is shown in Figure 5. The 4.8-kb HindIII DNA fragment, including the entire rat MLC2 gene (Nudel et al. 1984), was inserted into the HindIII site of plasmid $\mathrm{pCV}$, containing the chimeric actin-globin gene. The chimeric actin-globin gene contains about two-thirds of the $5^{\prime}$ region of the rat skeletal muscle actin gene plus $730 \mathrm{bp}$ of its $5^{\prime}$-flanking sequences spliced to about onethird of the 3 ' end of the human embryonic $\epsilon$-globin gene (Melloul et al. 1984). Both genes are in the same tran- 

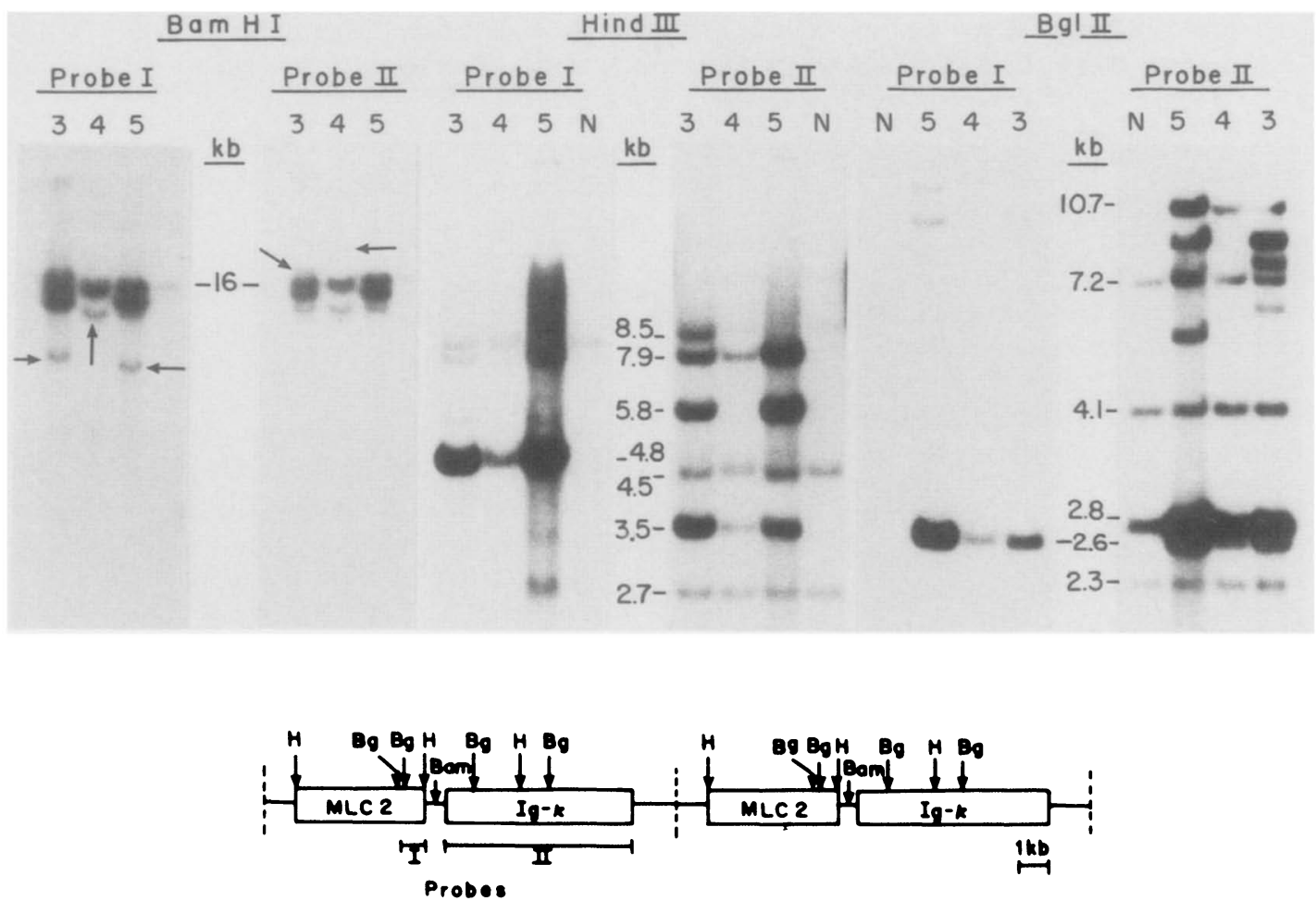

Figure 4. The organization of the exogenous MLC2 and the $\kappa$ genes in the genome of the LCk transgenic strains. Tail DNA (10 $\mu$ ) from transgenic strains LCk3, LCk4, and LCk5 was digested with BamHI, HindIII, or BglII, and the fragments were blot-hybridized to ${ }^{32}$ P-labeled probe, as described (Shani et al. 1984). (probe I) A 1-kb DNA fragment derived from the 5 ' end of the rat MLC2 gene; (probe II) a 7-kb fragment carrying the rearranged immunoglobulin gene. The arrows mark junction fragments containing inserted and host DNA, indicating a single site of integration in each transgenic strain. (Bottom) Location of restriction sites within plasmid pMLC-k that were used in the analysis and the probes used. (Bam) BamHI; $(H)$ HindIII; $(B g)$ BglII.

scriptional orientation. Plasmid pCV-LC was linearized before injection by digestion with the restriction enzyme Sall, which cuts once within the pBR322 sequences. Of the 19 mice born, four carried the injected DNA.

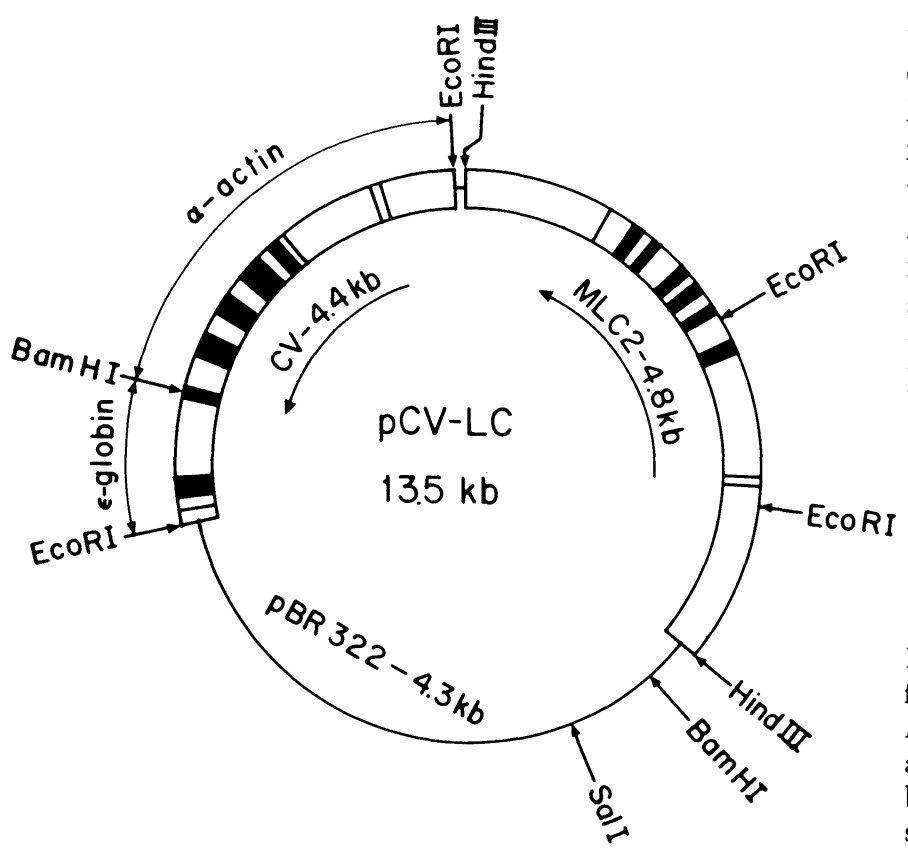

\section{Expression of the rat $M L C 2$ and the chimeric} actin-globin genes

RNA was prepared from skeletal and cardiac muscles and from several other tissues of offspring of the four transgenic strains. The presence of the rat MLC2 gene transcripts in the RNA preparations was determined by the RNase protection assay, as described above. In mice of the transgenic strains CVLC3 and CVLC4, no rat MLC2 transcripts could be detected in RNA prepared from striated muscles or from any of the other tissues tested. However, in offspring of mouse CVLC1, rat MLC2 transcripts were specifically found in skeletal muscle but not in cardiac muscle (even after long exposures of the autoradiograms) nor in the other tissues tested. The tissue-specific expression was stable for at least three generations (Fig. 6).

Figure 5. Map of plasmid pCV-LC. The 4.8-kb HindIII-HindIII fragment containing the rat MLC2 gene was inserted into the HindIII site of plasmid pCV containing the rat skeletal muscle actin/human $\epsilon$-globin chimeric gene. (Solid boxes) Exons; (open boxes) introns and flanking sequences. The direction of transcription is indicated by arrows. 
The presence of the chimeric actin-globin gene transcripts was determined by an S1 endonuclease assay, using the 187-bp DdeI DNA fragment derived from the human $\epsilon$-globin region of the construct as a probe (Melloul et al. 1984; Shani 1986). As observed for the rat MLC2 gene, no transcripts could be detected in mice of the transgenic strains CVLC3 and CVLC4. However, specific transcripts were detected in skeletal, as well as in cardiac, muscles of mouse strain CVLCl (Fig. 7). The level of the chimeric gene transcripts in skeletal muscle was about three times higher than that in cardiac muscle. This tissue specificity is consistent with the expression of the endogenous skeletal muscle actin gene (Minty et al. 1982; Mayer et al. 1984; Shani 1986).

In RNA prepared from the cardiac and skeletal muscles of the founder of a fourth transgenic strain, CVLC2, the actin-globin chimeric gene was expressed in the skeletal and cardiac muscles, whereas the MLC2 gene was expressed in the skeletal muscle only /data not shown). This mouse transmitted the exogenous genes to its offspring with normal Mendelian segregation. Southern blot analysis showed no difference in the pattern of the hybridizing fragments between the founder and its progeny over three generations (data not shown). However, no transcripts of the exogenous genes were detected in the heart and skeletal muscle, nor in other tissues, of the four transgenic offspring tested. The results for one of these mice are shown in Figures 6 and 7 . A similar phenomenon was observed by Palmiter et al. (1982) in transgenic mice carrying a metallothioneinthymidine kinase chimeric gene.

We showed previously that similar to the endogenous skeletal muscle actin gene (Minty et al. 1982; Mayer et al. 1984), the chimeric actin-globin gene underwent a transition in the relative tissue specificity during the development of four transgenic strains (Shani 1986). In neonatal mice the expression of the chimeric gene was higher in cardiac muscle than in skeletal muscle, whereas in adult mice it was higher in skeletal muscle. Figure 8 (left) shows a similar developmental transition in the expression of the chimeric actin-globin gene in mouse strain CVLC1. However, it is of interest that in neonatal mice of this transgenic strain, no rat MLC2 transcripts could be detected in the skeletal muscle, whereas the endogenous gene is expressed in this tissue (Fig. 8, right).

Figure 6. RNase mapping of the rat MLC2 transcripts in tissues of offspring of transgenic strains carrying plasmid pCVLC. Total RNAs $(10 \mu \mathrm{g})$ from the indicated tissues of offspring of transgenic mice strains CVLC1 and CVLC2, and from skeletal muscle of a control normal mouse and differentiated cultures of the rat myogenic cell line L8 (L8 post) were hybridized with the SP6 RNA probe, as described in the legend to Fig. 3. The first three lanes demonstrate the stable inheritance of expression of the exogenous gene in the skeletal muscle of the founder (SK.GO) in first-generation (SK.G1) and second-generation (Sk.G2) mice of the transgenic strain CVLC1. (Bottom) See details in the legend to Fig. 3.
Linkage between the exogenous rat $M L C 2$ and the chimeric actin-globin genes in the transgenic strain CVLC1

Figure 9 shows the Southern blot analysis of genomic DNA of mice of the CVLCl and CVLC2 strains. The EcoRI- and BamHI-digested DNAs were hybridized to the plasmid DNA carrying the rat MLC2 gene, whereas the SspI-digested DNAs were hybridized to the CV-LC plasmid. BamHI cuts the construct twice, generating the expected $8.3-$ and $5.3-\mathrm{kb}$ major bands. EcoRI cuts twice in the rat MLC2 and twice at the edges of the actinglobin gene, generating major bands of 5.3, 2.2, and 1.6 $\mathrm{kb}$. (The 4.4-kb band of the actin-globin gene was not labeled because the probe did not contain the relevant homologous sequences.) SspI cuts the construct once, generating the $13.5-\mathrm{kb}$ major fragment. In each of the three digests of CVLCI DNA, there are only two minor bands (indicated by asterisks) that do not have counterparts in the DNA of the control mouse. These results indicate that in the strain CVLCl, the exogenous DNA integrated at a single chromosomal site, all copies are found in a head-to-tail tandem array, and no detectable rearrangements occurred. In contrast, CVLC2 DNA has a few more minor bands, some of them more intense than the minor bands seen in CVLC1 DNA. As all tested transgenic progeny of this strain had the same pattern of hybridizing bands as the founder (data not shown), it appears that all of the copies of the injected plasmid inte-

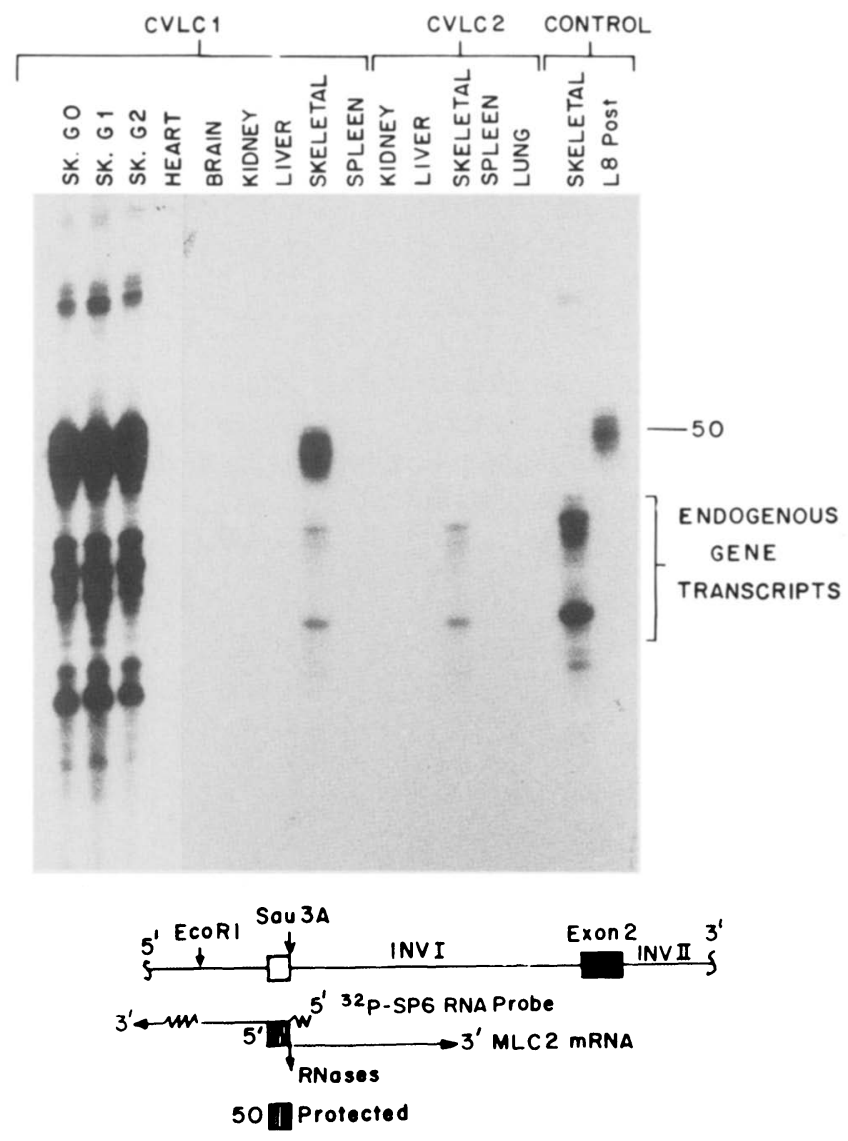




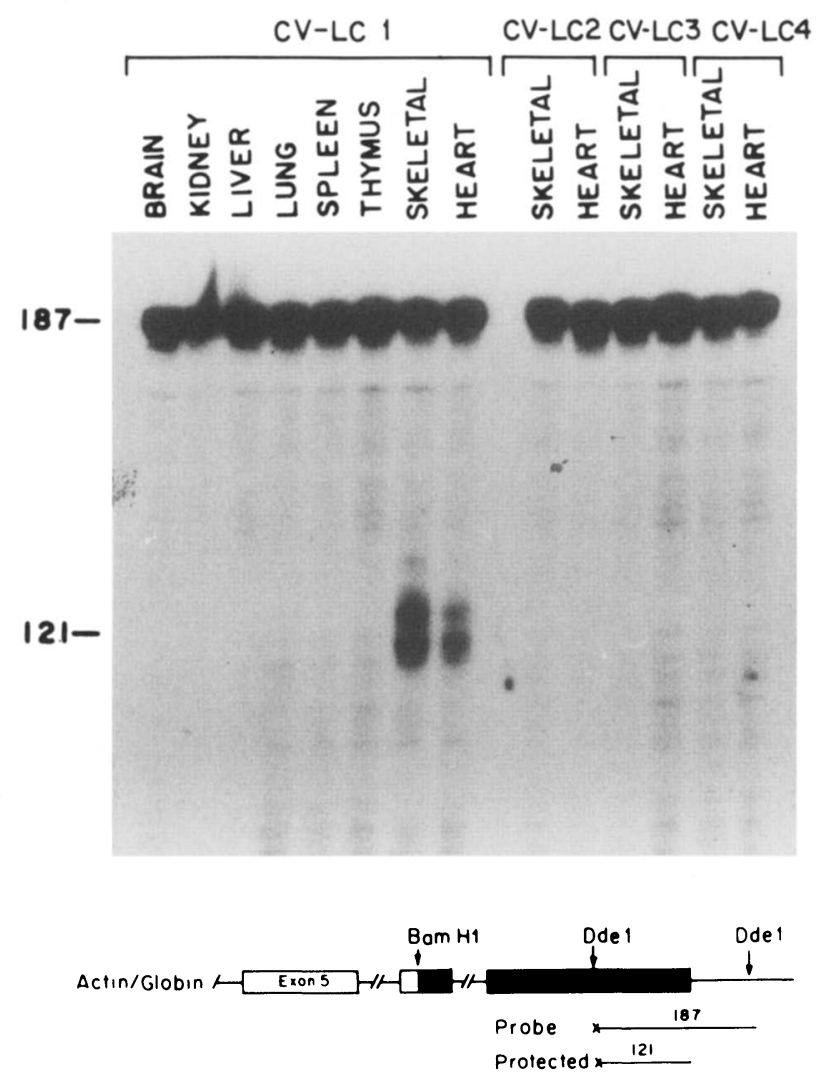

Figure 7. S1 analysis of actin-globin gene transcripts in tissues of transgenic strains CVLC1, CVLC2, CVLC3, and CVLC4 carrying plasmid pCV-LC. Total RNAs $(10 \mu \mathrm{g})$ from the indicated tissues were hybridized with a 187-bp DdeI DNA fragment derived from the human $\epsilon$-globin region of the construct. (Bottom) (Solid box) Human $\epsilon$-globin-coding sequences; (open box) actin-coding sequences. The 3' label is indicated by an asterisk. The numbers indicate the length of the probe and the protected fragment (nucleotides).

grated into one chromosome. The more intense minor bands most probably indicate that rearrangements occurred in a number of copies. We estimate the number of integrated plasmid copies to be about 5 and 10 for strains CVLC1 and CVLC2, respectively. Strains CVLC3 and CVLC4 each contain about 20 copies, with no detectable rearrangements (data not shown).

\section{Discussion}

The present investigation shows that two genes of different tissue specificity are able to maintain their appropriate patterns of expression when linked in close proximity and integrated into the same chromosomal site. Thus, in a transgenic mouse strain carrying the rearranged immunoglobulin $\kappa$ light chain and the rat MLC2 genes, the immunoglobulin gene was expressed specifically in the spleen, whereas the rat MLC2 gene was expressed in skeletal muscle only. Similarly, in the offspring of one transgenic strain and in the founder of another strain, both carrying the chimeric actin-globin and the rat MLC2 genes, the chimeric gene was ex- pressed in skeletal as well as in cardiac muscles, whereas the rat MLC2 gene was expressed in skeletal muscle only. In addition, this pair of genes was also differentially controlled during development with a pattern similar to that of the endogenous skeletal muscle actin gene. Expression of the actin-globin gene was higher in cardiac muscle than in the skeletal muscle of neonatal mice, whereas in adult mice it was higher in the skeletal muscle. These results therefore demonstrate a very high degree of autonomy of the genes in their control of tissue and developmental specific expression. However, this does not exclude the possibility that the integration site may play a decisive role in other combinations of exogenous and endogenous DNA sequences.

The rat MLC2 and skeletal muscle actin genes contain enhancer elements in their 5'-flanking region (D. Melloul et al., unpubl.), whereas the immunoglobulin $\kappa$ gene contains an enhancer in an intron between the variablejoining $(V I)$ and constant $(C)$ regions (Queen and Baltimore 1983; Picard and Schaffner 1984). Both types of enhancers contribute to the tissue specificity of these genes, yet none of these enhancers exerted any detectable influence on the mode of expression of the neighboring genes. Recently, it was reported that the immunoglobulin $\kappa$ enhancer can exert its influence evenly over a broad domain and that tandem $\kappa$ promoters falling within this domain are activated independently, irrespective of whether they are located 1.7 or $7.7 \mathrm{~kb}$ away

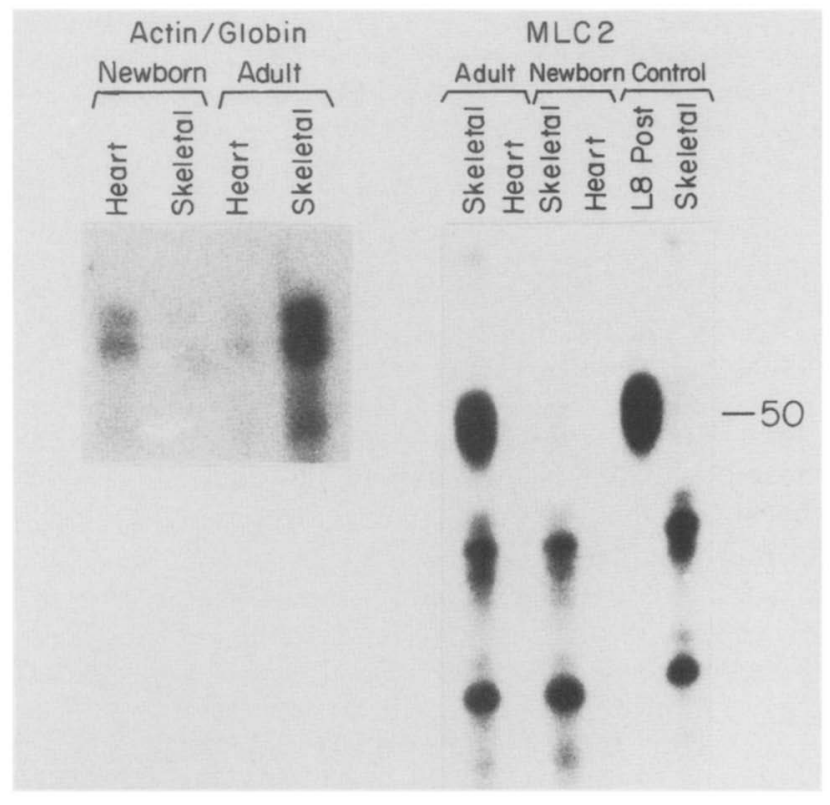

Figure 8. Rat MLC2 and actin-globin gene transcripts in striated muscles of newborn and adult mice of strain CVLC1 carrying both genes. Total RNAs $(10 \mu \mathrm{g})$ from skeletal and cardiac muscles of newborn and adult mice of strain CVLCl were hybridized either with the MLC2 riboprobe (right), as described in the legend to Fig. 3, or with the $\epsilon$-globin probe (left), as described in the legend to Fig. 7. RNA extracts from differentiated cultures of the rat myogenic cell line L8 (L8 post) and from normal skeletal muscle (skeletal) were used as controls. 


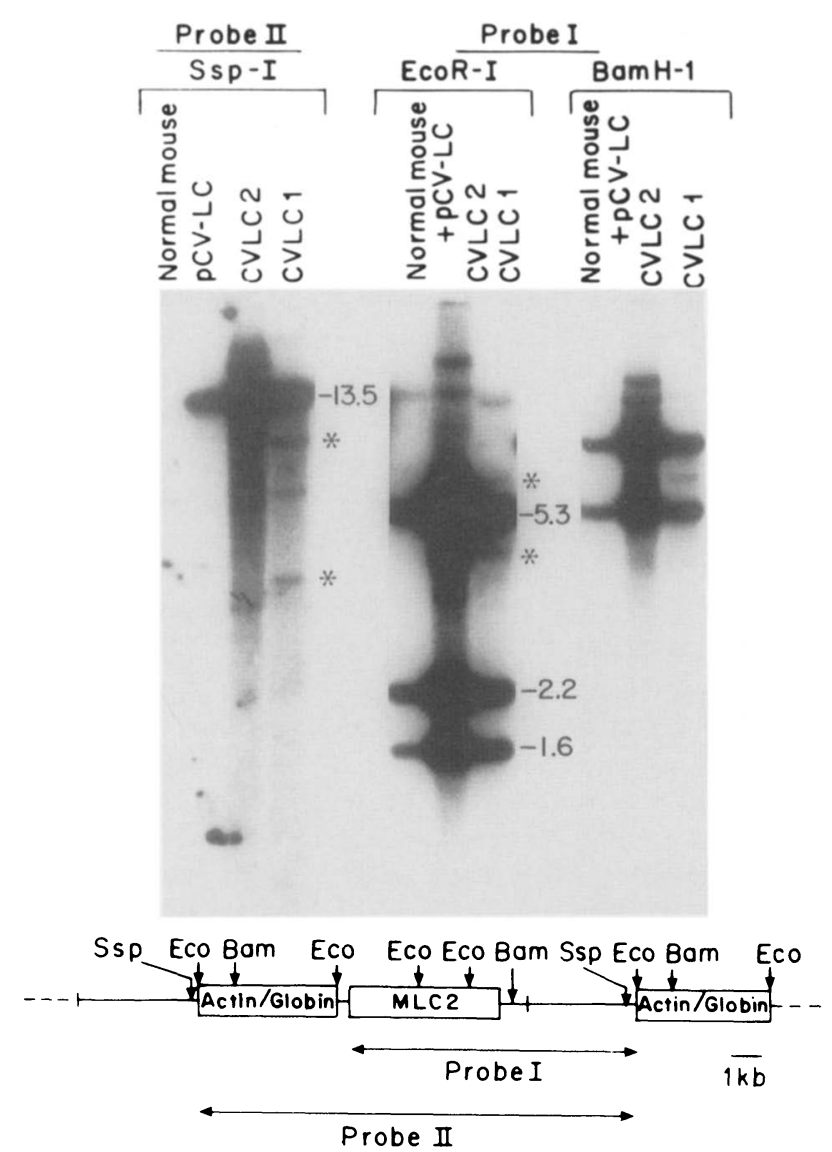

Figure 9. The organization of the exogenous MLC2 and the actin-globin genes in the genome of CVLC1 and CVLC2 transgenic strains. Ten micrograms of tail DNA from transgenic strains CVLC1 and CVLC2 and from a normal mouse DNA mixed with $100 \mathrm{pg}$ of plasmid pCV-LC were digested with BamHI, EcoRI, or SspI and blot-hybridized to ${ }^{32} \mathrm{P}$-labeled probes (Shani 1985). (Probe I) Plasmid pMLCH containing the rat MLC2 gene; (probe II) the entire pCV-LC plasmid. (*) Junction fragments containing inserted and host DNA, indicating a single site of integration in the transgenic strain CVLC1. (Bottom) Location of restriction sites within plasmid pCV-LC. Also indicated are the probes used. Short vertical bars indicate the sites where the plasmid was linearized prior to microinjection.

(Atchison and Perry 1986). It is therefore of interest that although the distance of the immunoglobulin gene enhancer from its own promoter or from the rat MLC2 promoter was about the same (3.6 and $3.8 \mathrm{~kb}$, respectively) in our construct, the enhancer had no detectable effect on the expression of the muscle-specific gene in three independent transgenic strains. It is possible that the $\kappa$ gene enhancer is more efficient at stimulating transcription from its own promoter than from a heterologous promoter. Recent studies using the immunoglobulin heavy-chain enhancer showed that this enhancer is not efficient when located some distance from a heterologous promoter (Wasylyk and Wasylyk 1986). In the case of transgenic strain LCk4, Southern blot analysis has shown clearly that the foreign genes are present in two or three copies per genome in a head-to-tail order, with no visible rearrangements of the inserted constructs. Thus, even if only the two distal genes are active in their respective tissues (with a distance of $20-36 \mathrm{~kb}$ between the two promoters), the fact remains that the promoters of both genes are adjacent to the enhancer sequences of an entirely different tissue specificity.

Activation of eukaryotic genes is associated with a reorganization of chromatin into a transcriptionally active conformation. The size of the chromosomal domain that is involved in the expression of genes has not yet been defined. DNase I-sensitivity mapping of several genes suggests that the active region extends from 12 to $100 \mathrm{~kb}$ (Lawson et al. 1982; Alevy et al. 1984; Forrester et al. 1986; Jantzen et al. 1986; Smith et al. 1986). The domain of increased sensitivity includes the structural genes and large portions of their $5^{\prime}$ - and 3'-flanking regions (Stadler et al. 1980; Weintraub et al. 1981). Whether the differential expression of two genes with different specificities that are found at the same chromosomal site reflects the formation of an active chromatin conformation restricted to the expressed gene only, or whether activation of each gene leads to the formation of an extended DNase I-sensitive domain that includes both genes remains to be determined. Experiments addressing this question in the $\beta$-globin locus indicated that all globin genes are contained within a DNase I-sensitive domain in erythroid tissue, and each of these genes is probably differentially activated during development by stagespecific factors as yet unknown (Weintraub and Groudine 1976; Miller et al. 1978; Lachman and Mears 1983).

In two out of the three transgenic strains containing the immunoglobulin-MLC2 construct, the rat MLC2gene transcripts could not be detected in any tissue while the immunoglobulin gene was expressed specifically in lymphoid tissues. Offspring of three out of the four mice injected with the MLC2-actin-globin genes did not express either gene. However, since a similar variability of expression was also obtained when plasmids containing a single gene were microinjected (Chada et al. 1985; Shani 1985; Townes et al. 1985a; Hammer et al. 1986; Krumlauf et al. 1986; Shani and Yaffe 1986), these results do not necessarily indicate an effect of the neighboring gene. Rather, they suggest that even in this regard (i.e., stably inherited differences in expression between the transgenic strains), each gene behaves independently of its neighboring gene. Considerable variation in the frequency and the level of expression among different transgenic Drosophila strains carrying identical P-element constructs has also been observed (Goldberg et al. 1983; Richards et al. 1983; Spradling and Rubin 1983; Hazelrigg et al. 1984).

After the present experiments were initiated, it became evident that the vector DNA sequences have an inhibitory effect on the expression of genes introduced into the germ line (Chada et al. 1985; Townes et al. 1985a; Krumlauf et al. 1986; Shani 1986). It is possible that the inhibitory effect of the pBR322 sequences contributed to the variability in the expression of the MLC2 and the actin-globin genes in our experiments.

Townes et al. (1985b) produced transgenic mice carrying the human $\beta$-globin gene and the mouse metal- 
lothionein-growth hormone fusion gene (MThGh) juxtaposed in opposite, head-to-head orientation. In most of their transgenic mice, expression of the $\beta$-globin gene revealed the same tissue specificity as that of the MThGh gene and was undetectable in erythroid tissues. In one transgenic mouse, there were indications that the human $\beta$-globin gene affected the expression of the MThGh gene. It is possible that placing the two genes in opposite orientation, head-to-head, brought the weak control sequences of the human $\beta$-globin gene under the direct control of the strong metallothionein enhancer. Because the $\beta$-globin enhancer is located 3' to the gene, it is conceivable that the metallothionein enhancer dominated the adjacent globin promoter. It would be of interest to test whether insertion of the rat MLC2 gene adjacent in opposite orientation, head-to-head to the chimeric actin-globin gene or the immunoglobulin gene would change the mode of expression of these genes.

\section{Materials and methods}

DNA constructions

Plasmid pMLC-k A 7-kb EcoRI-BamHI fragment, containing the rearranged mouse immunoglobulin $\kappa$ light-chain gene from myeloma MOPC41 (a gift from P. Leder), was filled in with Klenow fragment of DNA polymerase followed by ligation into the filled-in and dephosphorylated SalI site of plasmid pMLCH, containing the rat MLC2 gene in a $4.8-\mathrm{kb}$ insert (Nudel et al. 1984). The orientation of the immunoglobulin gene was determined by HindIII digestion.

Plasmid $p C V$-LC The 4.8-kb HindIII-HindIII fragment containing the rat MLC2 gene was ligated into the HindIII site of plasmid pCV, containing the rat $\alpha$-actin-human $\epsilon$-globin chimeric gene (Melloul et al. 1984). The orientation of the rat MLC2 gene was determined by EcoRI digestion.

\section{Production of transgenic mice}

Plasmids pMLC-k and pCV-LC were linearized with NdeI and SalI, respectively. They were then diluted to concentration of $10 \mu \mathrm{g} / \mathrm{ml}$ in $10 \mathrm{~mm}$ PIPES (pH 7.0), $150 \mathrm{mM} \mathrm{KCl}$, and $5 \mathrm{~mm}$ $\mathrm{NaCl}$.

Fertilized eggs were obtained from superovulated females of $(\mathrm{BALB}) / \mathrm{c} \times \mathrm{C} 57 \mathrm{BL} / 6 \mathrm{~J}) \mathrm{F}_{1}, 1$ day after mating with males of $(\mathrm{C} 57 \mathrm{BL} / 6) \times \mathrm{DBA} / 2 \mid \mathrm{F}_{1}$. After microinjection, the surviving eggs were implanted into the oviducts of pseudopregnant CD1 females.

\section{DNA isolation and analysis}

Genomic DNA was isolated from mouse tail by incubating 2-cm tail samples overnight at $55^{\circ} \mathrm{C}$ in $50 \mathrm{~mm}$ Tris $(\mathrm{pH} 8.0), 100$ mM EDTA, $0.1 \%$ SDS, and $200 \mu \mathrm{g} / \mathrm{ml}$ proteinase $\mathrm{K}$, followed by phenol/chloroform extractions.

Genomic DNA samples were digested with the appropriate restriction enzymes, separated on $1 \%$ agarose gels, transferred to nitrocellulose filters, and hybridized to nick-translated probes, as described (Shani et al. 1984). After hybridization, the filters were washed at $70^{\circ} \mathrm{C}$ in $0.1 \times$ SSC, $0.1 \%$ SDS, and $0.1 \%$ sodium pyrophosphate and exposed to Kodak XAR films.

\section{RNA isolation and analysis}

Tissues were frozen in liquid nitrogen, and RNA was prepared by the lithium chloride-urea extraction method (Auffray and Rougeon 1980). RNase protection analysis was performed as described (Melton et al. 1984). The RNA probe was derived from an SP6 plasmid subclone containing a 290-bp EcoRI-Sau3A DNA fragment, which includes the first exon and the 5'flanking region of the rat MLC2 gene (Nudel et al. 1984; Shani 1985; Shani and Yaffe 1986). Samples were hybridized at $55^{\circ} \mathrm{C}$, followed by digestion with RNase at $37^{\circ} \mathrm{C}$ for $1 \mathrm{hr}$. Sl protection analysis with end-labeled DNA probes was performed as described (Berk and Sharp 1977). Hybridization was at $48^{\circ} \mathrm{C}$ both for the immunoglobulin $\kappa$ probe (Bergman et al. 1984) and the $\epsilon$-globin probe (Shani 1986). Protected fragments were analyzed on polyacrylamide/urea sequencing gels.

\section{Acknowledgments}

We thank Ms. Ola Yoffe for excellent technical assistance and Dr. Rebecca Aft for a critical reading of the manuscript. This work was supported by National Institutes of Health grant GM-22767, the Muscular Dystrophy Association (USA), The Israel Academy of Sciences and Humanities, and by the United States-Israel Binational Agricultural Research and Development Fund (BARD).

\section{References}

Alevy, M.C., M.J. Tsai, and B.W. O'Malley. 1984. DNase I sensitive domain of the gene coding for the glycolytic enzyme glyceraldehyde-3-phosphate dehydrogenase. Biochemistry 23: 2309-2314.

Auffray, C. and F. Rougeon. 1980. Purification of immunoglobulin heavy chain mRNA from total myeloma tumor RNA. Eur. J. Biochem. 107: 303-313.

Babinet, C., H. Farza, D. Morello, M. Hadchouri, and C. Pourcell. 1985. Specific expression of hepatitis B surface antigene (HBsAG) in transgenic mice. Science 230: 1160-1163.

Bergman, Y., D. Rice, R. Grosschedl, and D. Baltimore. 1984. Two regulatory elements for immunoglobulin $\kappa$ light chain expression. Proc. Natl. Acad. Sci. 81: 7041-7045.

Berk, A.J. and P.A. Sharp. 1977. Sizing and mapping of early adenovirus mRNA by gel electrophoresis of S1 endonuclease-digested hybrids. Cell 12: 721-732.

Boshart, M., F. Weber, G. Jahn, B. Dorsch-Hasler, B. Fleckenstein, and W. Schaffner. 1984. A very strong enhancer located upstream of an immediate early gene of human cytomegalovirus. Cell 41: 521-530.

Brinster, R.L., K.A. Ritchie, R.E. Hammer, R.L. O'Brien, B. Arp, and U. Storb. 1983. Expression of a microinjected immunoglobulin gene in the spleen of transgenic mice. Nature 306: $332-336$.

Cattanach, B.M. 1974. Position effect variegation in the mouse. Genet. Res. 23: 291-306.

Chada, K., J. Magram, K. Raphael, G. Radice, E. Lacy, and F. Costantini. 1985. Specific expression of a foreign $\beta$-globin gene in erythroid cells of transgenic mice. Nature 314: 300 380.

Chao, M.V., P. Mellon, P. Charnay, T. Maniatis, and R. Axel. 1983 . The regulated expression of $\beta$-globin genes introduced into mouse erythroleukemia cells. Cell 32: 483-493.

Charnay, P. R. Treisman, P. Mellon, M. Chao, R. Axel, and T. Maniatis. 1984. Differences in human $\alpha$ and $\beta$ globin gene expression in mouse erythroleukemia cells: The role of intragenic sequences. Cell 38: 251-263. 
Forrester, W.C., C. Thompson, I.T. Elder, and M. Groudine. 1986. A developmentally stable chromatin structure in the human $\beta$-globin gene cluster. Proc. Natl. Acad. Sci. 83: $1359-1369$.

Goldberg, D.A., J.W. Posakony, and T. Maniatis. 1983. Correct developmental expression of a cloned alcohol dehydrogenase gene transduced into the Drosophila germ-line. Cell 34: $59-73$.

Grosschedl, R., D. Weaver, D. Baltimore, and F. Costantini. 1984. Introduction of a $\mu$ immunoglobulin gene into the mouse germ-line: Specific expression in lymphoid cells and synthesis of functional antibody. Cell 38: 647-658.

Hammer, R.E., R.L. Brinster, and R.D. Palmiter. 1986. Use of gene transfer to increase animal growth. Cold Spring Harbor Symp. Quant. Biol. 50: 379-387.

Hazelrigg, T., R. Levis, and G.M. Rubin. 1984. Transformation of white locus DNA in Drosophila: Dosage compensation, zeste interaction, and position effect. Cell 36: 469-481.

Jantzen, K., H.P. Fritton, and T. Igo-Kemenes. 1986. The DNase I sensitive domain of the chicken lysozyme gene spans 24 kb. Nucleic Acids Res. 14: 6085-6099.

Klein, G. and E. Klein. 1985. Evolution of tumors and the impact of molecular oncology. Nature 316: 190-198.

Kondoh, H., K. Yasuda, and J.J. Okada. 1983. Tissue specific expression of a cloned chick $\delta$-crystallin gene in transgenic mice. Nature 301: 440-442.

Krumlauf, R., R.E. Hammer, S.M. Tilghtman, and R.L. Brinster. 1986. Developmental regulation of $\alpha$-fetoprotein genes in transgenic mice. Mol. Cell. Biol. 5: 1639-1648.

Lacy, E., S. Roberts, P. Evans, M.D. Burtenshaw, and F. Costantini. 1983. A foreign $\beta$-globin gene in transgenic mice: Integration at abnormal chromosomal positions and expression in inappropriate tissues. Cell 34: 343-358.

Lawson, G.M., B.J. Knoll, C.J. March, S.L.C. Woo, M.J. Tsai, and B.J. O'Malley. 1982. Definition of 5' and 3' structural boundaries of the chromatin domain containing the ovalbumin multigene family. J. Biol. Chem. 257: 1501-1507.

Mayer, Y., H. Czosnek, P.E. Zeelon, D. Yaffe, and U. Nudel. 1984. Expression of the genes coding for the skeletal muscle and cardiac actins in the heart. Nucleic Acids Res. 12: $1087-1100$.

Melloul, D., B. Aloni, J. Calvo, D. Yaffe, and U. Nudel. 1984. Developmentally regulated expression of chimeric genes containing muscle actin DNA sequences in transfected myogenic cells. EMBO I. 3: 983-990.

Melton, D.A., P.A. Krieg, M.R. Rebagliati, T. Maniatis, T. Zinn, and M.R. Green. 1984. Efficient in-vitro synthesis of biologically active RNA and RNA hybridization probes from plasmids containing a bacteriophage SP6 promoter. Nucleic Acids Res. 12: 7035-7056.

Miller, D.M., P. Turner, A.W. Nienhuis, D.E. Axelrod, and T.V. Gopalakrishnan. 1978. Active conformation of the globin genes in uninduced and induced mouse erythroleukemia cells. Cell 14: $511-521$.

Minty, A.J., S. Alonso, M. Cavarotti, and M.B. Buckingham. 1982. A fetal skeletal muscle actin mRNA in the mouse and its identity with cardiac actin mRNA. Cell 30: 185-192.

Nudel, U., J. Calvo, M. Shani, and Z. Levy. 1984. The nucleotide sequence of a rate myosin light chain 2 gene. Nucleic Acids Res. 12: 7175-7186.

Palmiter, R.D., H.Y. Chen, and R.L. Brinster. 1982. Differential regulation of metallothionein-thymidine kinase fusion genes in transgenic mice and their offspring. Cell 29: $701-$ 710.

Picard, D. and W. Schaffner. 1984. A lymphocyte-specific enhancer in the mouse immunoglobulin $\kappa$ gene. Nature 307: $80-82$.
Queen, C. and D. Baltimore. 1983. Immunoglobulin gene transcription is activated by downstream sequence element. Cell 33: 741-748.

Renkawitz, R., H. Beng, T. Graf, P. Mathias, M. Grez, and G. Schutz. 1982. Expression of a chicken lysozyme recombinant gene is regulated by progesterone and dexamethasone after microinjection into oviduct cells. Cell 31: 167-176.

Richards, G., A. Cassab, M. Bouvouis, D. Jarry, and C. Dissons. 1983. The normal developmental regulation of a cloned sgs 3' "glue" gene chromosomally integrated in Drosophila by P-element transformation. EMBO I. 2: 2137-2142.

Scott, R.W., T.F. Vogt, M.E. Croke, and S.M. Tilghman. 1984. Tissue-specific activation of a cloned $\alpha$-fetoprotein gene during differentiation of a transfected embryonal carcinoma cell line. Nature 310: $562-567$.

Shani, M. 1985. Tissue-specific expression of rat myosin light chain 2 gene in transgenic mice. Nature 314: 283-286.

- 1986. Tissue-specific and developmentally regulated expression of a chimeric actin/globin gene in transgenic mice. Mol. Cell. Biol. 6: 2624-2631.

Shani, M. and D. Yaffe. 1986. Expression of muscle specific genes in transgenic mice. In Molecular biology of muscle development (ed. C. Emerson, D.A. Fischman, B. NadalGinard, and M.A.Q. Siddiqui), UCLA Symposium on Molecular and Cellular Biology, New Series, vol. 29, pp. 575-590, Alan R. Liss, New York.

Shani, M., S. Admon, and D. Yaffe. 1984. The methylation state of 2 muscle-specific genes: Restriction enzyme analysis did not detect a correlation with expression. Nucleic Acids Res. 12: $7225-7234$.

Smith, R.D., J. Yu, A. Annunziato, and R.L. Seale. 1984. Chromatin structure of the $\beta$-globin gene family in murine erythroleukemia cells. Biochemistry 23: 2970-2976.

Spofford, J. 1976. Position-effect vaiegation in Drosophila. In The genetics and biology of Drosophila (ed. M. Ashburner, and E. Novitski), pp. 955-1018. Academic Press, London.

Spradling, A.C. and G.M. Rubin. 1983. The effect of chromosomal position on the expression of the Drosophila xanthine dehydrogenase gene. Cell 34: 47-57.

Storb, U., R.L. O'Brien, M.D. McMullen, K.A. Gollahon, and R.L. Brinster. 1984. High expression of a cloned immunoglobulin $\kappa$ gene in transgenic mice is restricted to B lymphocytes. Nature 310: 238-241.

Townes, T.M., J.B. Lingrel, H.Y. Chen, R.L. Brinster, and R.D. Palmiter. 1985a. Erythroid specific expression of human $\beta$ globin genes in transgenic mice. EMBO /. 4: 1715-1723.

Townes, T.M., H.Y. Chen, J.B. Lingrel, R.D. Palmiter, and R.L. Brinster. 1985b. Expression of human $\beta$-globin genes in transgenic mice: Effects of a flanking metallothioneinhuman growth hormone fusion gene. Mol. Cell. Biol. 5: $1977-1983$.

Wasylyk, C. and B. Wasylyk. 1986. The immunoglobulin heavy chain B lymphocyte enhancer efficiently stimulates transcription in non-lymphoid cells. EMBO I. 5: 553-560.

Weintraub, H. and M. Groudine. 1981. $\alpha$-Globin gene switching during the development of chicken embryos: Expression and chromosome structure. Cell 24: 333-344.

Weintraub, H. and M. Groudine. 1976. Chromosomal subunits in active genes have an altered conformation. Science 193: $848-856$. 


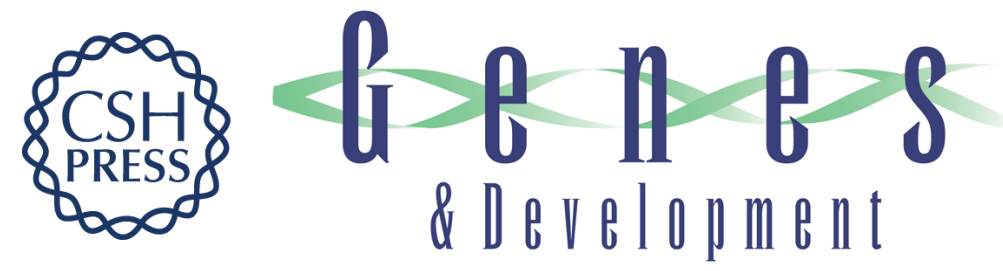

\section{Expression in transgenic mice of two genes of different tissue specificity integrated into a single chromosomal site.}

P Einat, Y Bergman, D Yaffe, et al.

Genes Dev. 1987, 1:

Access the most recent version at doi:10.1101/gad.1.10.1075

References This article cites 45 articles, 7 of which can be accessed free at:

http://genesdev.cshlp.org/content/1/10/1075.full.html\#ref-list-1

License

Email Alerting Receive free email alerts when new articles cite this article - sign up in the box at the top Service right corner of the article or click here.

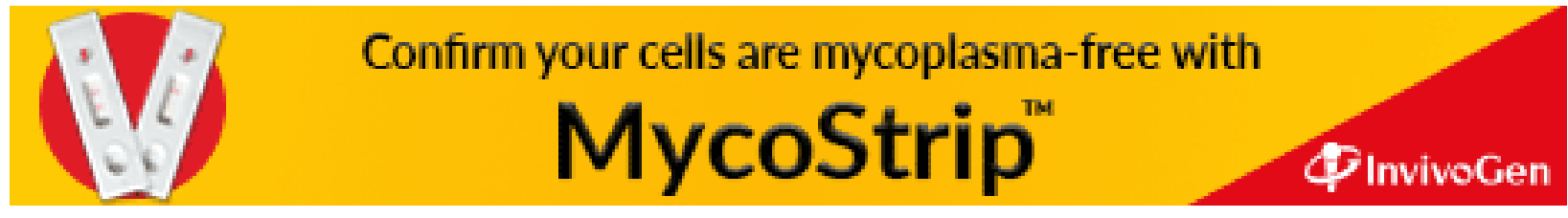

